\title{
The heterogeneity of cancer
}

\author{
H. Gilbert Welch ${ }^{1}$
}

Received: 22 January 2018 / Accepted: 23 January 2018 / Published online: 2 February 2018

(c) Springer Science+Business Media, LLC, part of Springer Nature 2018

Cancer used to be so simple. It started as a wayward cell that then underwent a stepwise progression: from in situ to local, local to regional and, finally, regional to distant disease. At least, that is what I was taught in medical school...some (gulp) 40 years ago.

Narod and Sopik suggest a wildly different paradigm. Local growth and distant metastasis are independent phenomena. Local control of cancer (e.g., efforts to minimize local recurrence) has no effect on its tendency to metastasize. If a cancer is destined to spread to distant sites, it will have already done so.

Call it the "bad cancers are bad" model. Or, alternatively, "good cancers are good."

Oddly enough, in 1955 a cancer surgeon at the Cleveland clinic_-George Crile Jr.-foretold this complexity on the pages of Life magazine:

In clinical practice to say that a person has cancer gives as little information about the possible course of his disease as to say that he has an infection. There are dangerous infections that may be fatal and there are harmless infections that are self-limited or may disappear. The same is true of cancers. Cancer is not a single entity. It is a broad spectrum of diseases related to each other only in name. [1]

Narod and Sopik are not suggesting that size, stage, and nodal status are unassociated with the propensity to metastasize, rather that we have gotten the direction of causality wrong. The conventional model has been that large tumors are more likely to metastasize because they have a large pool of cancer cells to disseminate. Narod and Sopik instead suggest that these tumors became large because they are more aggressive cancers and thus are more likely to metastasize.

H. Gilbert Welch

gil.welch@dartmouth.edu

1 The Dartmouth Institute for Health Policy and Clinical Practice, Hanover, NH, USA
Large, late-stage, node positive lesions are simply valuable markers for "badness."

The corollary is that small, early-stage, node negative lesions are valuable markers for "goodness." But not always. Which brings us to the conundrum of DCIS.

It would be simplest if all DCIS was pseudodiseasecancer not destined to ever cause problems for our patients. Most DCIS is pseudodisease, but as Narod documented in earlier work [2], about $3 \%$ of women with DCIS will die from breast cancer in the next 20 years. Over half of these women did not experience an in-breast invasive recurrence prior to death.

In other words, bad breast cancers are bad-from the get go.

This phenomenon explains the limited ability of mammography to reduce breast cancer mortality. The lack of value in finding microscopic breast cancers (like DCIS) is one of the least well-recognized findings from the ten randomized trials of mammography. Only one trial addressed this important question, the second Canadian trial [3]. The control group received an annual clinical breast exam: a standardized, thorough (5-15 min per patient) physical exam of the breast generally done by specially trained nurses. The intervention group received the same thorough clinical exam each year plus a mammogram. In other words, Canada 2 tested the additional value of detecting abnormalities that cannot be felt. Given the finding of no difference in breast cancer mortality between the two groups, the lesson is clear: there is no obvious value to finding breast cancers that are so small they cannot be felt (such as most DCIS).

Overdiagnosis is made possible by cancers at the other end of the spectrum. Overdiagnosis is the detection of cancers that are very good-so good that patients would be better off not having them detected. Overdiagnosis doesn't limit the ability of mammography to reduce breast cancer mortality-instead it's a side-effect of the effort.

Such heterogeneity in cancer poses huge challenges for our effort to catch the disease early. It's been described as the "barnyard pen of cancers" (an analogy that likely originates with Crile). We are trying to catch birds, rabbits, and turtles. 
We can't catch the birds early, because they have already gone- these are the most aggressive cancers, those that have already spread by the time they are detectable. We are able to catch the rabbits- the more slowly progressive cancersbut their earlier detection may not help much, because they weren't destined to metastasize anyway. And then there are the turtles. There's no need catch them, because they're not going anywhere anyway.

\section{Compliance with ethical standards}

Conflict of interest Dr. Welch declares he has no conflict of interest.

Ethical approval This article does not contain any studies with human participants or animals performed by author.

\section{References}

1. Crile G (1955) A plea against the blind fear of cancer. An experienced surgeon says that excessive worry leads to costly tests, undue suffering and unnecessary operations. Life Mag 128-142

2. Narod SA, Iqbal J, Giannakeas V, Sopik V, Sun P (2015) Breast cancer mortality after a diagnosis of ductal carcinoma in situ. JAMA Oncol 1:888-896

3. Miller AB, To T, Baines CJ, Wall C (2000) Canadian National Breast Screening Study-2: 13-year results of a randomized trial in women aged 50-59 years. J Natl Cancer Inst 92(18):1490-1499 